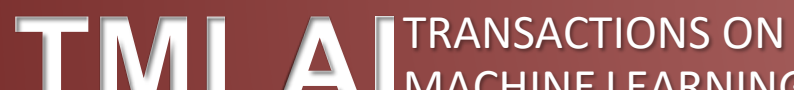

\section{Artificial Human Optimization - An Overview}

\author{
Satish Gajawada \\ Alumnus, Indian Institute of Technology Roorkee \\ Founder, Artificial Human Optimization - A New Field \\ gajawadasatish@gmail.com \\ Hassan M. H. Mustafa \\ Faculty of Specified Education, Dept. of Educational Technology, Banha University, Egypt \\ prof.dr.hassanmoustafa@gmail.com
}

\begin{abstract}
The main idea to author this article is to "Popularize Artificial Human Optimization Field like never before by showing an Overview of this new field". This idea can be divided into following sub-ideas:

1) To show the definition of "Artificial Human Optimization Field (AHO Field)"

2) To show difficulty level of creating new algorithms under AHO field

3) To show 30+ titles of papers published under AHO field

4) To show names of $65+$ authors who worked under AHO Field

5) To show best negative reviews obtained for work under AHO Field

6) To show best positive reviews obtained for work under AHO Field

7) To show feedback given by an expert for work under AHO Field

8) To show "Hassan Satish Particle Swarm Optimization (HSPSO)". This is latest work under AHO Field

9) To show contribution of Satish Gajawada and co-authors to this new Field

10) To show surprising results obtained after implementing $A H O$ algorithms

11) To show you "Future of Artificial Human Optimization Field"
\end{abstract}

\section{Sub-idea 1: Definition}

Artificial Human Optimization (AHO) is a new field proposed in December 2016. This work was published in Transactions on Machine Learning and Artificial Intelligence. All optimization methods which were proposed based on Artificial Humans will come under the new field titled Artificial Human Optimization [1]. The first paper in AHO field was proposed in 2006 [2].

\section{Sub-idea 2: Difficulty Level}

The following is the review obtained from an expert in 2013 for a work in AHO Field:

"The motivation of the paper is interesting. But the paper does not present any evaluation of the proposed algorithm. So we have an idea but we are not able to assess it on the basis of the paper. Next, there seems to be a difference between birds, fishes, ants, bacteria, bees etc. on one side, and human beings on the 
Satish Gajawada; Hassan M. H. Mustafa; Artificial Human Optimization - An Overview, Transactions on Machine Learning and Artificial Intelligence, Volume 6 No 4 August (2018); pp: 21-29

other side. Birds, fishes, ants, bacteria, bees etc. are more or less the same. People are different. I dare say that taxi drivers are different from politicians, or preschool teachers for example. Some people prefer money or power than love. It is not so difficult to guess which way ants will go but it is not so obvious when we consider people behavior. In my opinion the paper is a very first step to build the algorithm assumed but still lots of work is needed to achieve the goal."

From the above review it is clear that optimization methods based on Humans is not as easier as developing optimization methods based on Birds, fishes, ants, bacteria, bees etc.

\section{Sub-idea 3: Titles of Papers}

The following are the titles of papers published under AHO Field according to [3]:

1) Human behavior-based optimization: a novel metaheuristic approach to solve complex optimization problems

2) Human Behavior Algorithms for Highly Efficient Global Optimization

3) Human Behavior-Based Particle Swarm Optimization

4) POSTDOC : THE HUMAN OPTIMIZATION

5) Focus Group: An Optimization Algorithm Inspired by Human Behavior

6) ENTREPRENEUR : Artificial Human Optimization

7) Modification of particle swarm optimization with human simulated property

8) Human cognition inspired particle swarm optimization algorithm

9) Human-inspired algorithms for continuous function optimization

10) Anarchic Society Optimization: A human-inspired method

11) The Human-Inspired Algorithm: A Hybrid Nature-Inspired Approach to Optimizing Continuous Functions with Constraints

12) CEO: Different Reviews on PhD in Artificial Intelligence

13) Artificial Human Optimization - An Introduction

14) An Ocean of Opportunities in Artificial Human Optimization Field

15) 25 Reviews on Artificial Human Optimization Field for the First Time in Research Industry

16) A New Optimization Method Based on Adaptive Social Behavior: ASBO

17) Human meta-cognition inspired collaborative search algorithm for optimization

18) Self regulating particle swarm optimization algorithm

19) Improved SRPSO algorithm for solving CEC 2015 computationally expensive numerical optimization problems

20) Clustering of Text Document based on ASBO

21) PID Controller Auto tuning using ASBO Technique 
22) ASBO Based Compositional in Combinatorial Catalyst

23) Seeker Optimization Algorithm

24) Teaching-learning-based optimization: A novel method for constrained mechanical design optimization problems

25) Imperialist competitive algorithm: An algorithm for optimization inspired by imperialistic competition

26) Group Counseling Optimization: A Novel Approach

27) A Simple Human Learning Optimization Algorithm

28) A novel optimization algorithm inspired by the creative thinking process

29) Immigrant Population Search Algorithm for Solving Constrained Optimization Problems

30) Democracy-inspired particle swarm optimizer with the concept of peer groups

31) Social Emotional Optimization Algorithm for Nonlinear Constrained Optimization Problems

32) Human opinion dynamics: An inspiration to solve complex optimization problems

\section{Sub-idea 4: Names of authors}

The following are the researchers who made their contribution to AHO Field according to [3]:

1) Satish Gajawada

2) Hassan M. H. Mustafa

3) Seyed-Alireza Ahmadi

4) Da-Zheng Feng

5) Han-Zhe Feng

6) Hai-Qin Zhang

7) Hao Liu

8) Gang Xu

9) Gui-yan Ding

10) Yu-bo Sun

11) Edris Fattahi

12) Mahdi Bidar

13) Hamidreza Rashidy Kanan

14) Ruo-Li Tang

15) Yan-Jun Fang

16) Muhammad Rizwan Tanweer 
17) Suresh Sundaram

18) L. M. Zhang

19) C. Dahlmann

20) Y. Zhang

21) A. Ahmadi-Javid

22) Mingyi Zhang, Luna

23) Zhang, Yanqing

24) Manoj Kumar Singh

25) N. Sundararajan

26) Prakasha $S$

27) H R Shashidhar

28) G T Raju

29) Sridhar N

30) Nagaraj Ramrao

31) Manoj Kumar Singh

32) Devika P. D

33) Dinesh P. A

34) Rama Krishna Prasad

35) Chaohua Dai

36) Yunfang Zhu

37) Weirong Chen

38) R.V.Rao

39) V.J.Savsani

40) D.P.Vakharia

41) Esmaeil Atashpaz-Gargari

42) Caro Lucas

43) M. A. Eita

44) M. M. Fahmy

45) Ling Wang

46) Haoqi $\mathrm{Ni}$ 
47) Ruixin Yang

48) Minrui Fei

49) Wei Ye

50) Xiang Feng

51) Ru Zou

52) Huiqun $Y u$

53) Hamid Reza Kamali

54) Ahmad Sadegheih

55) Mohammad Ali Vahdat-Zad

56) Hassan Khademi-Zare

57) Ritambhar Burman

58) Soumyadeep Chakrabarti

59) Swagatam Das

60) Yuechun Xu

61) Zhihua Cui

62) Jianchao Zeng

63) Rishemjit Kaur

64) Ritesh Kumar

65) Amol P. Bhondekar

66) Pawan Kapur

\section{Sub-idea 5: Best Negative Reviews}

The following are the best negative reviews obtained for work under AHO field:

1) This paper studies a so-called human optimization method which falls into the research topic of optimization. The proposed method was presented on the first page followed by some discussions. The paper clearly makes no novel contribution to the state of the art on optimization algorithms and techniques. Thus, because of this lack of new contribution, the paper is not appropriate for the conference.

2) Nothing to evaluate

3) Funny paper, especially the notion of "love array" :)

4) This is not a research paper. It should not have been submitted for review. Rationale and results are completely lacking. I do not even think there is a research idea in there. 


\section{Sub-idea 6: Best Positive Reviews}

The following are the best positive reviews obtained for work under AHO field:

1) We had a glance at your published article "POSTDOC : THE HUMAN OPTIMIZATION". We found your article very innovative, insightful and interesting. We really value your outstanding contribution towards Scientific Community.

2) Very Interesting (from IEEE TAAI 2013)

3) Very Novel (from Springer SOCTA 2017)

4) Very Impressive

5) Compelling and Creative (from experts of aitoday.xyz)

6) New and Interesting Area of Research (from world class conference PAKDD 2018)

\section{Sub-idea 7: Feedback received by Satish Gajawada}

Below is the feedback from an expert when Satish Gajawada (Founder, Artificial Human Optimization) asked to give feedback on work under AHO:

"Thanks for the message. It seems you are the "father of Artificial Human Optimization" field, it will be tomfoolery on my part to provide feedback on such topic. You are already at the zenith of this research."

\section{Sub-idea 8: Latest Work}

Hassan Satish Particle Swarm Optimization (HSPSO) is the latest work under AHO Field. It is shown below: HSPSO is obtained by incorporation of MSHO concepts into Particle Swarm Optimization. In starting and even generations the Artificial Humans move towards the best fitness value. In odd generations Artificial Humans move away from the worst fitness value. In HSPSO, we maintain local worst of particle and global worst of all particles in addition to local best of particle and global best of all particles. This is shown in lines 4 to 17 . In lines 19 to 24 velocity is calculated by moving towards the local best of particle and global best of all particles. In lines 26 to 31 pseudo code for odd generations is shown in below text. In these odd generations particles move away from local worst of particle and also away from global worst of all particles. In line 33, number of iterations is incremented by one. Then control goes back to line number 4. This process of moving towards the best in one generation and moving away from the worst in next generation is continued until termination criteria has been reached.

Procedure: Hassan Satish Particle Swarm Optimization ( HSPSO )

1) Initialize all particles

2) iterations $=0$

3) do

4) for each particle i do

5) If $\left(f\left(x_{i}\right)<f\left(\right.\right.$ pbest $\left.\left._{i}\right)\right)$ then

6) $\quad$ pbest $_{i}=x_{i}$

7) end if

8) if $\left(f\left(\right.\right.$ pbest $\left._{i}\right)<f($ gbest $\left.)\right)$ then

9) $\quad$ gbest $=$ pbest $_{\mathrm{i}}$

10) end if 


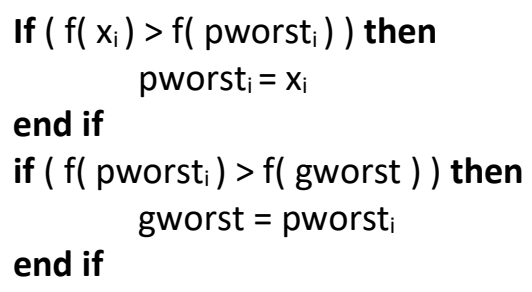

34) while ( termination condition is false)

\section{Sub-idea 9: Contribution of Satish Gajawada and Co-authors}

1) Entrepreneur: Artificial Human Optimization. Transactions on Machine Learning and Artificial Intelligence, Volume 4 No 6 December (2016); pp: 64-70.

2) CEO: Different Reviews on PhD in Artificial Intelligence, Global Journal of Advanced Research, vol. 1, no.2, pp. 155-158, 2014.

3) POSTDOC : The Human Optimization, Computer Science \& Information Technology (CS \& IT), CSCP, pp. 183-187, 2013.

4) Artificial Human Optimization - An Introduction, Transactions on Machine Learning and Artificial Intelligence, Volume 6, No 2, pp: 1-9, April 2018.

5) An Ocean of Opportunities in Artificial Human Optimization Field, Transactions on Machine Learning and Artificial Intelligence, Volume 6, No 3, June 2018.

6) 25 Reviews on Artificial Human Optimization Field for the First Time in Research Industry, International Journal of Research Publications, Volume 5, No 2, United Kingdom, 2018.

7) Collection of Abstracts in Artificial Human Optimization Field, International Journal of Research Publications, Volume 7, No 1, United Kingdom, 2018.

8) HIDE : Human Inspired Differential Evolution - An Algorithm under Artificial Human Optimization Field, International Journal of Research Publications (Volume: 7, Issue: 1), http://ijrp.org/paper_detail/264

9) Hybridization concepts of Artificial Human Optimization field Algorithms incorporated into Particle Swarm Optimization (In Progress)

10) Artificial Human Optimization - An Overview 
Satish Gajawada; Hassan M. H. Mustafa; Artificial Human Optimization - An Overview, Transactions on Machine Learning and Artificial Intelligence, Volume 6 No 4 August (2018); pp: 21-29

11) An Artificial Human Optimization Algorithm titled Human Thinking Particle Swarm Optimization (In Progress)

12) Testing Multiple Strategy Human Optimization based Artificial Human Optimization Algorithms (In Progress)

\section{Sub-idea 10: Surprising Results}

In [10], PSO method performed well than the proposed HSPSO method for particular parameter settings and benchmark function. But the general expectation is that after adding Artificial Human Optimization concepts into PSO, the proposed HSPSO method should perform well. But this is not the case.

\section{Sub-idea 11: Future}

According to [7], there exists millions of opportunities in AHO Field. Some interesting opportunities possible in near Future are shown below:

1) International Institute of Artificial Human Optimization, Hyderabad, INDIA

2) Indian Institute of Technology Roorkee Artificial Human Optimization Labs, IIT Roorkee

3) Foundation of Artificial Human Optimization, New York, USA.

4) IEEE Artificial Human Optimization Society

5) ELSEVIER journals in Artificial Human Optimization

6) Applied Artificial Human Optimization - A New Subject

7) Advanced Artificial Human Optimization - A New Course

8) Invited Speech on "Artificial Human Optimization" in world class Artificial Intelligence Conferences

9) A Special issue on "Artificial Human Optimization" in a Springer published Journal

10) A Seminar on "Recent Advances in Artificial Human Optimization" at Technical Festivals in colleges

\section{REFERENCES}

[1] Satish Gajawada; Entrepreneur: Artificial Human Optimization. Transactions on Machine Learning and Artificial Intelligence, Volume 4 No 6 December (2016); pp: 64-70

[2] Dai C., Zhu Y., Chen W. (2007) Seeker Optimization Algorithm. In: Wang Y., Cheung Y., Liu H. (eds). Computational Intelligence and Security. CIS 2006. Lecture Notes in Computer Science, vol 4456. Springer, Berlin, Heidelberg.

[3] Satish Gajawada and Hassan M. H. Mustafa, "Collection of Abstracts in Artificial Human Optimization Field", International Journal of Research Publications, Volume 7, No 1, United Kingdom, 2018.

[4] Satish Gajawada, "CEO: Different Reviews on PhD in Artificial Intelligence", Global Journal of Advanced Research, vol. 1, no.2, pp. 155-158, 2014.

[5] Satish Gajawada, "POSTDOC : The Human Optimization", Computer Science \& Information Technology (CS \& IT), CSCP, pp. 183-187, 2013.

[6] Satish Gajawada, "Artificial Human Optimization - An Introduction", Transactions on Machine Learning and Artificial Intelligence, Volume 6, No 2, pp: 1-9, April 2018. 
[7] Satish Gajawada, "An Ocean of Opportunities in Artificial Human Optimization Field", Transactions on Machine Learning and Artificial Intelligence, Volume 6, No 3, June 2018.

[8] Satish Gajawada, "25 Reviews on Artificial Human Optimization Field for the First Time in Research Industry", International Journal of Research Publications, Volume 5, No 2, United Kingdom, 2018.

[9] Satish Gajawada, Hassan M. H. Mustafa, HIDE : Human Inspired Differential Evolution - An Algorithm under Artificial Human Optimization Field, International Journal of Research Publications (Volume: 7, Issue: 1), http://ijrp.org/paper_detail/264

[10] Satish Gajawada and Hassan M. H. Mustafa, Hybridization concepts of Artificial Human Optimization field Algorithms incorporated into Particle Swarm Optimization (In Progress)

[11] Satish Gajawada and Hassan M. H. Mustafa, Artificial Human Optimization - An Overview.

[12] Satish Gajawada and Hassan M. H. Mustafa, An Artificial Human Optimization Algorithm titled Human Thinking Particle Swarm Optimization (In Progress).

[13] Satish Gajawada and Hassan M. H. Mustafa, Testing Multiple Strategy Human Optimization based Artificial Human Optimization Algorithms (In Progress). 